\title{
A Mixed Convection Boundary Layer Flow over a Vertical Wall in a Porous Medium, with Exponentially Varying Fluid Viscosity
}

\author{
Eunice Mureithi \\ Department of Mathematics, University of Dar es Salaam, Dar es Salaam, Tanzania \\ Email: ewambui02@gmail.com
}

Received 20 May 2014; revised 18 June 2014; accepted 3 July 2014

Copyright (C) 2014 by author and Scientific Research Publishing Inc.

This work is licensed under the Creative Commons Attribution International License (CC BY).

http://creativecommons.org/licenses/by/4.0/

(c) () Open Access

\section{Abstract}

This study investigates a mixed convection boundary layer flow over a vertical wall embedded in a highly porous medium. The fluid viscosity is assumed to decrease exponentially with temperature. The boundary layer equations are transformed into a non-similar form using an appropriate nonsimilar variable $\xi$ and a pseudo-similar variable $\eta$. The non-similar equations are solved using an efficient local non-similarity method. The effect of viscosity variation parameter on the heat transfer, skin friction and the velocity and temperature distribution within the boundary layer is investigated. The viscosity variation parameter, the viscous dissipation parameter and non-similarity variable are shown to have a significant effect on velocity and thermal boundary layer and also on the skin friction coefficient and heat transfer at the wall.

\section{Keywords}

Porous Medium, Non-Darcy, Mixed Convection Boundary Layer, Temperature Dependent Viscosity

\section{Introduction}

Mixed convection boundary layer flow through a porous medium is of primary importance due to its applications in industry such as geothermal operations (for example dynamics of hot springs), terrestrial heat flow through an aquifer, flow of moisture through porous industrial material etc. Engineering applications include porous thermal insulation and heat exchangers with fluidized beds etc.

Reviews on convective heat transfer in porous medium are well documented in the books by Nield and Bejan, [1] and Pop and Ingham [2], Kaviany [3] and in the handbook of porous media edited by Vafai [4]. Numerous studies on mixed convection boundary through a porous media using the Darcy model and extended Darcy 
models have been carried out, by others, by Karabis et al. [5] investigated a mixed forced-free convection boundary layer flow over a vertical wall in a highly porous medium, Chen [6] who looked at a boundary layer flow over a horizontal in a saturated porous medium. Using the model developed by Vafai and Tien [7], Hong et al. [8] investigated the non-Darcian effects on a flow over a vertical plate in porous media.

Most fluids used in engineering have temperature varying viscosity. A study by Hossain and Munir [9] investigated a mixed convection boundary layer with temperature dependent viscosity where it was shown that the viscosity variation parameter has an effect on the wall skin friction coefficient and wall heat transfer coefficient. Jayanthi and Kumari [10] studied the effects of variable viscosity on a non-Darcy flow of a non-Newtonian fluid over a vertical surface in porous medium. They showed that variable viscosity parameter has a significant effect on the fluid flow properties.

The current study focuses on a boundary layer flow over a vertical wall embedded in a highly porous medium and with variable viscosity. The non-similar boundary layer equations are solved using the local non-similarity method due to Sparrow et al. [11] and Sparrow and Yu [12]. In Section 2 the mixed convection boundary layer flow equations are formulated and transformed to the non-similar form, in Section 3 the equations are solved and the results presented and discussed in Section 4. Conclusions are drawn in Section 5.

\section{Mixed Convection Boundary Layer Flow in a Porous Medium}

A steady, two-dimensional flow of a viscous incompressible fluid over a heated semi-infinite vertical wall embedded in a highly porous medium is studied. The $x$-axis is taken along the wall and the $y$-axis as the normal axis to $x$-axis. The free-stream velocity and temperature are taken as $U_{\infty}$ and $T_{\infty}$ respectively and the wall temperature as $T_{w}\left(>T_{\infty}\right)$. The fluid viscosity $\mu$ is assumed to decrease exponentially with temperature. Under the boundary layer approximation for a flow through a porous medium and the Boussinesq's approximation, the mixed convection boundary layer equations through a porous media take the form:

$$
\begin{gathered}
\frac{\partial u}{\partial x}+\frac{\partial v}{\partial y}=0 \\
\rho_{0}\left(u \frac{\partial u}{\partial x}+v \frac{\partial u}{\partial y}\right)=\rho_{0} U_{\infty} \frac{\mathrm{d} U_{\infty}}{\mathrm{d} x}+g \rho_{0} \alpha\left(T-T_{\infty}\right)+\frac{\tilde{\mu}}{K}\left(U_{\infty}-u\right)+\frac{\partial}{\partial y}\left(\tilde{\mu}(T) \frac{\partial u}{\partial y}\right) \\
\rho_{0} c_{p}\left(u \frac{\partial T}{\partial x}+v \frac{\partial T}{\partial y}\right)=\kappa \frac{\partial^{2} \mathrm{~T}}{\partial \mathrm{y}^{2}}+\tilde{\mu}(T)\left(\frac{\partial u}{\partial y}\right)^{2}
\end{gathered}
$$

The appropriate boundary conditions for the flow are:

$$
\begin{array}{ll}
u(x, 0)=v(x, 0)=0, & T(x, 0)=T_{w} \\
u(x, \infty)=U_{\infty}(x), & T(x, \infty)=T_{\infty}
\end{array}
$$

Here $g$ is the gravitational acceleration, $\alpha$ is the coefficient of thermal expansion, $\kappa$ is the thermal conductivity, $\quad c_{p}$ is the specific heat at constant pressure, $\rho_{0}$ is the fluid density at reference temperature $T_{0}$ and $K$ is the permeability of the porous medium. The wall temperature $T_{w}$ is assumed to be a constant. Most common fluids have dynamic viscosity that varies exponentially with temperature. Here we use Arrhenius model which takes the exponential form

$$
\tilde{\mu}(T)=\mu_{0} e^{-\gamma\left(T-T_{\infty}\right)}
$$

where $\mu_{0}$ is reference viscosity at reference temperature $T_{0}$ and $\gamma$ is a viscosity variation number defined as

$$
\gamma=\frac{1}{T_{w}-T_{\infty}} \ln \left(\frac{\mu_{0}}{\mu_{w}}\right)
$$

where $\mu_{0}>\mu_{w}$ with be $\mu_{w}$ being the fluid viscosity at the wall temperature $T_{w}$. For gases $\gamma<0$ and for liquids it is positive. For small values of $\gamma$ Taylor expansion leads to the linear or the inverse relation of viscosity with temperature. 


\section{Non-Similar Boundary Layer Equations}

The equations are rendered non-dimensional by introducing a boundary layer pseudo-similarity variable $\eta(x, y)$ and a non-similar variable $\xi(x)$, defined respectively as

$$
\eta(x, y)=\frac{y}{x} \sqrt{R e_{x}} \quad \xi(x)=\frac{G r_{x}}{R e_{x}^{2}}
$$

with $G r_{x}$ and $R e_{x}$ being the local Grash of number and the local Reynolds number, defined as

$$
G r_{x}=\frac{g \alpha\left(T_{w}-T_{\infty}\right) x^{3}}{v_{0}^{2}}, R e_{x}=\frac{U_{\infty} x}{v_{0}}
$$

where $v_{0}=\frac{\mu_{0}}{\rho_{0}}$ is the kinematic viscosity.

We use the scaling

$$
u=U_{\infty}(x) f^{\prime}(\eta, \xi), T-T_{\infty}=\left(T_{w}-T_{\infty}\right) \theta(\eta, \xi), \mu(\theta)=e^{-\varepsilon \theta},
$$

where $\mu(\theta)=\tilde{\mu} / \mu_{0}$ is the dimensionless viscosity and $\varepsilon=\gamma\left(T_{w}-T_{\infty}\right)$.

From the equation of continuity:

$$
v=-\frac{U_{\infty}}{\sqrt{R e_{x}}}\left[\frac{\beta+1}{2} f+\frac{\eta(\beta-1)}{2} f^{\prime}+\xi(1-2 \beta) f_{\xi}\right]
$$

with

$$
\beta=\frac{x}{U_{\infty}} \frac{\mathrm{d} U_{\infty}}{\mathrm{d} x}
$$

Assuming power law variation in the free-stream velocity $U_{\infty}=A x^{n}$, we have $\beta=n$. The non-similar boundary layer equations take the form:

$$
\begin{gathered}
f^{\prime \prime \prime}=\varepsilon \theta^{\prime} f^{\prime \prime}-S\left(1-f^{\prime}\right)+e^{\varepsilon \theta}\left[n\left[\left(f^{\prime}\right)^{2}-1\right]-\frac{n+1}{2} f f^{\prime \prime}-\xi \theta\right]+e^{\varepsilon \theta} \xi(1-2 n)\left[f_{\xi}^{\prime} f^{\prime}-f_{\xi} f^{\prime \prime}\right] \\
\theta^{\prime \prime}=-\operatorname{Pr}\left[\frac{n+1}{2} f \theta^{\prime}+E c\left(f^{\prime \prime}\right)^{2} e^{-\varepsilon \theta}\right]+\operatorname{Pr} \xi(1-2 n)\left[f^{\prime} \theta_{\xi}-f_{\xi} \theta^{\prime}\right]
\end{gathered}
$$

where $S_{x}$ is the dimensionless local permeability parameter, $\operatorname{Pr}$ is the Prandtl number and $E c$ is the local Eckert number, defined respectively as

$$
S_{x}=\frac{v_{0} X}{K U_{\infty}}, \quad \operatorname{Pr}=\frac{\mu_{0} c_{p}}{\kappa}, \quad E c_{x}=\frac{U_{\infty}^{2}}{c_{p}\left(T_{w}-T_{\infty}\right)} .
$$

The boundary conditions, in non-dimensional form, are:

$$
\begin{aligned}
& f^{\prime}(\xi, 0)=0, \quad(n+1) f(\xi, 0)+2 \xi(1-2 n) f_{\xi}(\xi, 0)=0, \quad \theta(\xi, 0)=1 \\
& \lim _{\eta \rightarrow \infty} f^{\prime}(\xi, \eta)=1, \quad \lim _{\eta \rightarrow \infty} \theta(\xi, \eta)=0 .
\end{aligned}
$$

The shear stress and the heat transfer at the wall can be represented using the local skin friction coefficient and the local Nusselt number, defined by

$$
c_{f} \sqrt{R e_{x}}=e^{-\varepsilon} f^{\prime \prime}(\xi, 0), \quad R e_{x}^{-\frac{1}{2}} N u=-\theta^{\prime}(\xi, 0)
$$

\section{Numerical Solutions of the Non-Similar Boundary Layer Equations}

The system of Equations (2.4)-(2.6) is solved using the non-similar method of Sparrow et al. [11]. This method has been used extensively in solving non-similar mixed convection boundary layer equations by among other 
Sparrow and Yu [12], Gorla et al. [13], Mureithi and Mason [14], Bég et al. [15] etc. The method involves approximating the solutions at different levels of truncation. We introduce the notation:

$$
f_{1}=\frac{\partial f}{\partial \xi}, f_{1}^{\prime}=\frac{\partial f^{\prime}}{\partial \xi}, \quad \theta_{1}=\frac{\partial \theta}{\partial \xi}, f_{2}=\frac{\partial f_{1}}{\partial \xi}, \theta_{2}=\frac{\partial \theta_{1}}{\partial \xi} \text { etc. }
$$

The first level of approximation involves solving the local similarity equations, where all terms involving the $\xi$ and its derivatives (that is the non-similar terms, $f_{1}, \theta_{1}$ ) in the Equations (2.4)-(2.6) are neglected.

At the second level of truncation, the method involves solving the system (2.4)-(2.6) together with the auxiliary system obtained by differentiating (2.4)-(2.6) partially with respect $\xi$ and neglecting all terms involving second order differentiation with respect to $\xi$ (that is $f_{2}, \theta_{2}$ etc. The auxiliary system takes the form:

$$
\begin{gathered}
f_{1}^{\prime \prime \prime}=\varepsilon\left(\theta_{1}^{\prime} f^{\prime \prime}+\theta^{\prime} f_{1}^{\prime \prime}\right)+S f_{1}^{\prime}+\varepsilon e^{\varepsilon \theta} \theta_{1}\left[n\left(\left(f^{\prime}\right)^{2}-1\right)-\frac{n+1}{2} f f^{\prime \prime}-\xi \theta\right]+\varepsilon e^{\varepsilon \theta} \xi(1-2 n)\left[f f_{1}^{\prime}-f_{1} f^{\prime \prime}\right] \\
+e^{\varepsilon \theta}\left[2 n f_{1}^{\prime}-\frac{n+1}{2}\left[f_{1} f^{\prime \prime}+f_{1}^{\prime \prime}\right]\right]-e^{\varepsilon \theta}\left[\xi \theta_{1}+\theta\right]+e^{\varepsilon \theta}(1-2 n)\left[f f_{1}^{\prime}-f_{1} f^{\prime \prime}+\xi\left[\left(f_{1}^{\prime}\right)^{2}-f_{1} f_{1}^{\prime \prime}\right]\right] \\
\frac{1}{\operatorname{Pr}} \theta_{1}^{\prime \prime}=-\frac{n+1}{2}\left(f_{1} \theta^{\prime}+f \theta_{1}^{\prime}\right)-E c e^{-\varepsilon \theta}\left[2 f_{1}^{\prime \prime}-\varepsilon\left(f^{\prime \prime}\right)^{2} \theta_{1}\right]+(1-2 n)\left[f^{\prime} \theta_{1}-\theta^{\prime} f_{1}+\xi\left[f_{1}^{\prime} \theta_{1}-f_{1} \theta_{1}^{\prime}\right]\right] \\
f_{1}^{\prime}(\xi, 0)=0, \quad[3(1-n)] f_{1}(\xi, 0)=0, \quad \theta_{1}(\xi, 0)=0, \quad f_{1}^{\prime}(\xi, \infty)=\theta_{1}(\xi, 0)=0
\end{gathered}
$$

The system (2.4)-(2.9) solved as if it is an ordinary differential system, with $\xi$ being treated as a parameter. The results obtained are a better approximation than those obtained at the first level of approximation.

An even better approximation can be obtained at the third order level of approximation, where the system (2.4)-(2.9) are solved together with an of auxiliary system obtained by further differentiating Equations (2.7)-(2.9) partially with respect to $\xi$ and all terms containing third order partials with respect to $\xi$ are neglected.

\section{Discussion of Results}

We approximate the solution of (2.4)-(2.6) using the second level of approximation. The system of Equations (2.4)-(2.9) is solved using the fourth order Runge-Kutta method combined with a Newton root refinement scheme. Computations were carried out for the case when $\mathrm{Pr}=0.7$ (air) and viscosity that increases exponentially with increase in temperature $(\varepsilon<0)$.

Figure 1 show the effect varying the non-similar parameter $\xi$ on the scaled stream-wise velocity, $f^{\prime}$, and viscosity, $\mu(\theta)$, distribution in the boundary layer. At a fixed stream-wise location, $\xi$ becomes a buoyancyrelated parameter. As $\xi$ increases velocity overshoots (exceeding free-stream velocity) are observed within the boundary layer. The maximum values of the super-velocities increase with increase in $\xi$ Results in figure 1also show that increasing $\xi$ has the effect of decreasing the thickness of the viscosity boundary layer.

The effect of varying the viscous dissipation parameter $E c$ was investigated and the results are presented in Figure 2. The results indicate that as $E c$ increases, the amplitude of the super-velocities increase. Temperature is also shown to increase with increase in $E_{c}$ and for high enough values of $E_{c}$ (in this case $E_{c} \geq 0.6$ ) there is a small region adjacent to the wall, where temperature exceeds its wall value of one. It follows that viscosity function also has positive gradient in that small region. This is an indication that increasing $E c$ results in increased heat transfer from the wall to the fluid.

Figure 3 shows stream-wise velocity and viscosity distributions within the boundary layer for different values of $\varepsilon$. The results show that at a fixed value of $\xi$ velocity overshoots increase with increase in $\varepsilon$. On the contrary, viscosity decreases with increase in $\varepsilon$.

Heat transfer at the wall (represented by the Nusselt number, $-\theta(\xi, 0)$ and shear stress at the wall (represented by skin friction coefficient, $\mathrm{e}^{-\varepsilon} f^{\prime \prime}(\xi, 0)$ were investigated as functions of the effect of $\xi$ and various values of $\varepsilon$ Figure 4 shows that the skin friction coefficient and Nusselt number increase with increase in $\varepsilon$. The skin friction coefficient increases linearly with $\xi$ while the Nusselt number decreases with increase in $\xi$.

Similar results showing the effect of $E_{c}$ on both the skin friction and the Nusselt number are presented in Figure 5. The results show that the skin friction increases linearly with increase in $\xi$ while the Nusselt number decrease with increase in $E c$. On the other hand, increase $E c$ results in increase in the skin friction coeffi- 


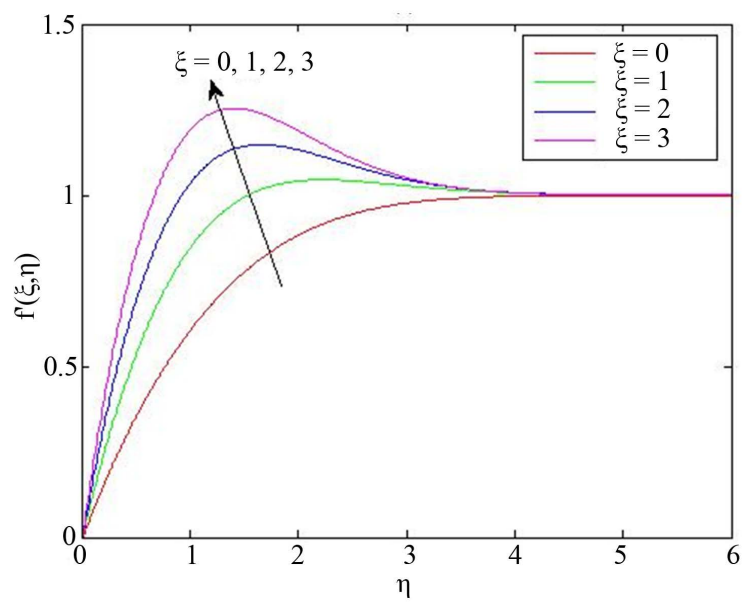

(a)

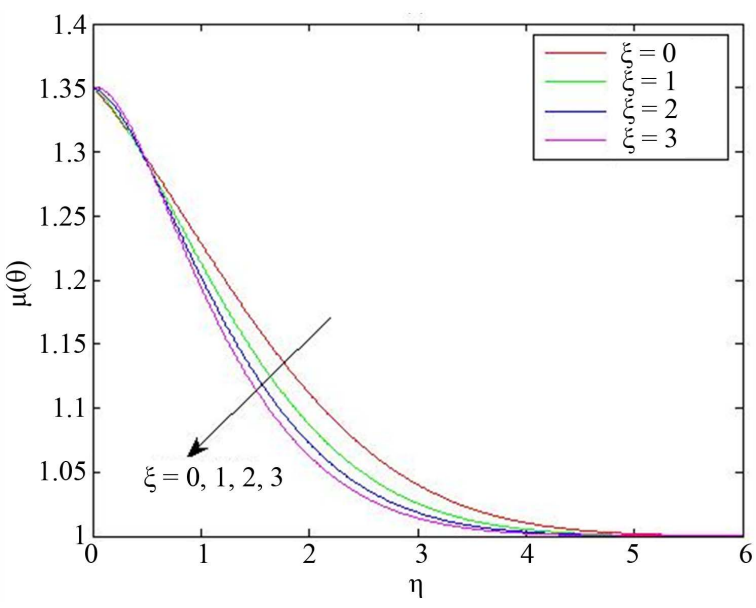

(b)

Figure 1. The effect of varying $\xi$ on (a) scaled stream-wise velocity $f^{\prime}$ and (b) viscosity $\mu(\theta)$ for the case when $E_{C}=0.4, S=0.5, n=0.2, \varepsilon=-0.3$.

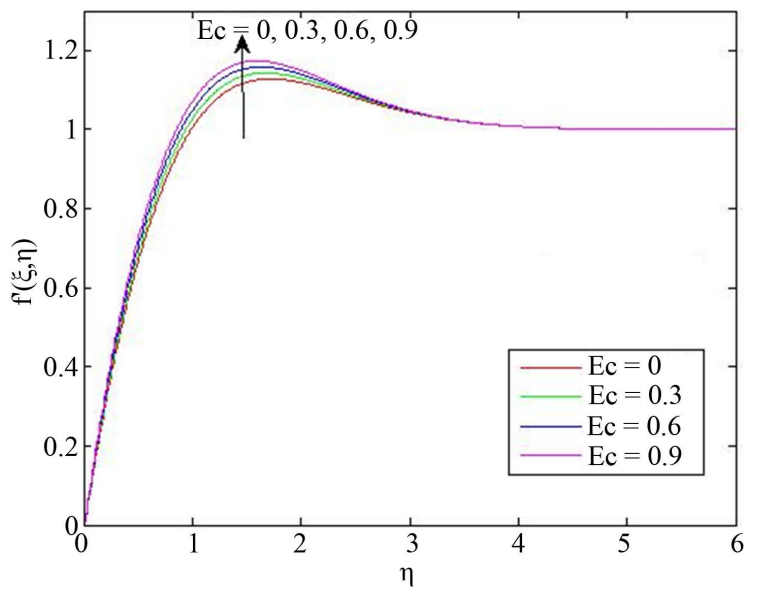

(a)

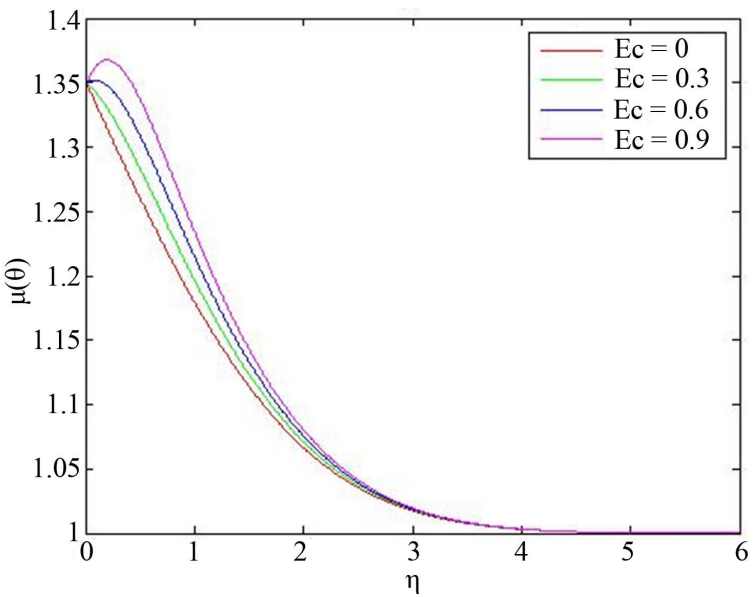

(b)

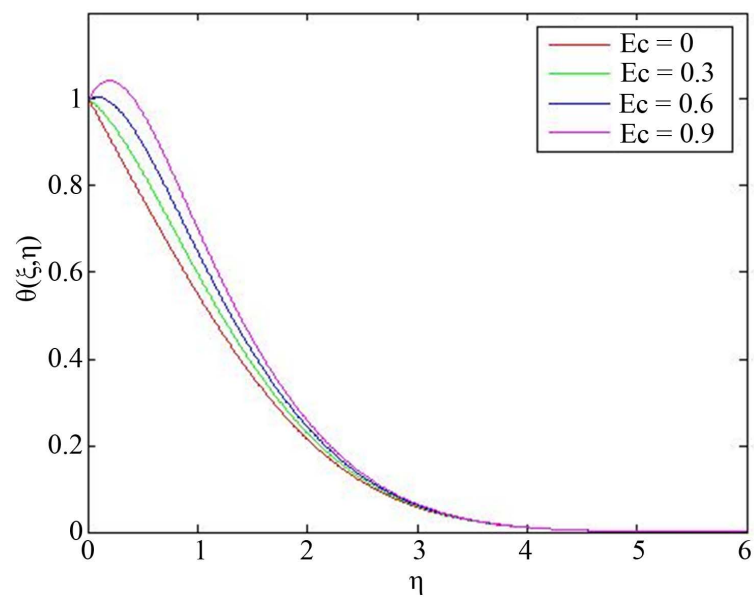

(c)

Figure 2. Effect of varying $E_{c}$ on (a) $f^{\prime}(\xi, \eta)$, (b) $\mu(\theta)$ and (c) $\theta(\xi, \eta)$ for the case when $\varepsilon=-0.3, S=0.5, n=0.2$, $\xi=2$. 


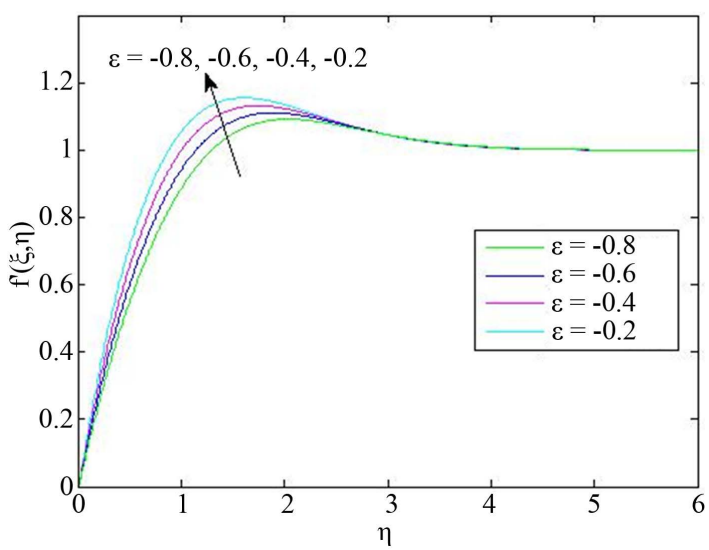

(a)

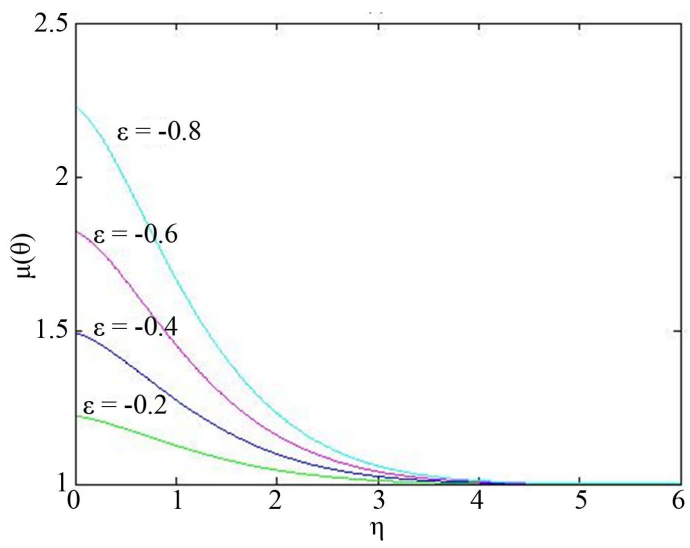

(b)

Figure 3. Effect of varying viscosity variation parameter $\varepsilon$ on (a) $f^{\prime}$ and (b) $\mu(\theta)$ for the case when $E c=0.4$, $S=0.5, \xi=2, n=0.2$.

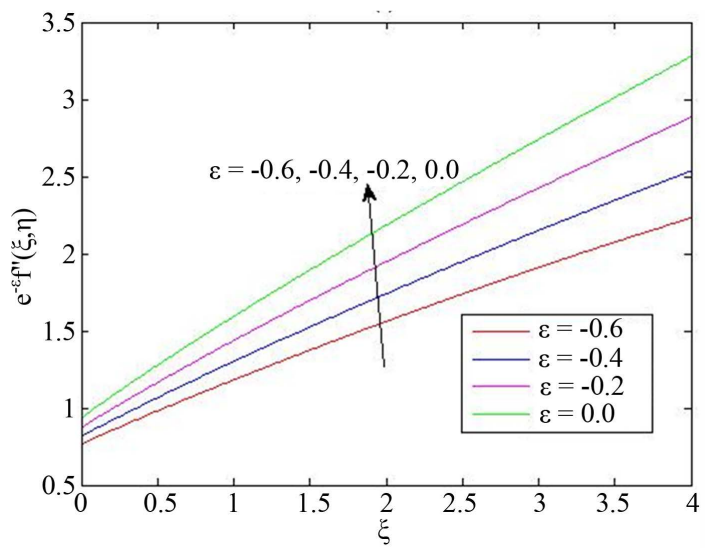

(a)

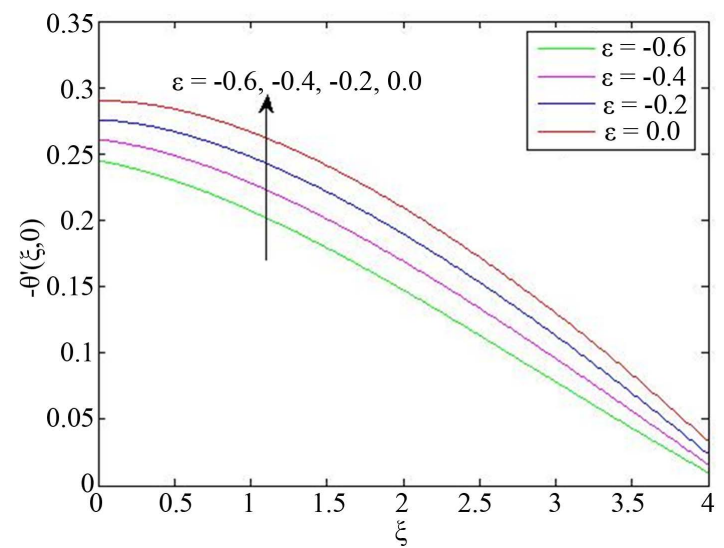

(b)

Figure 4. The effect of varying $\xi$ and $\varepsilon$ on the (a) skin friction coefficient and (b) Nusselt number for $E c=0.4$, $S=0.5, n=0.2$.

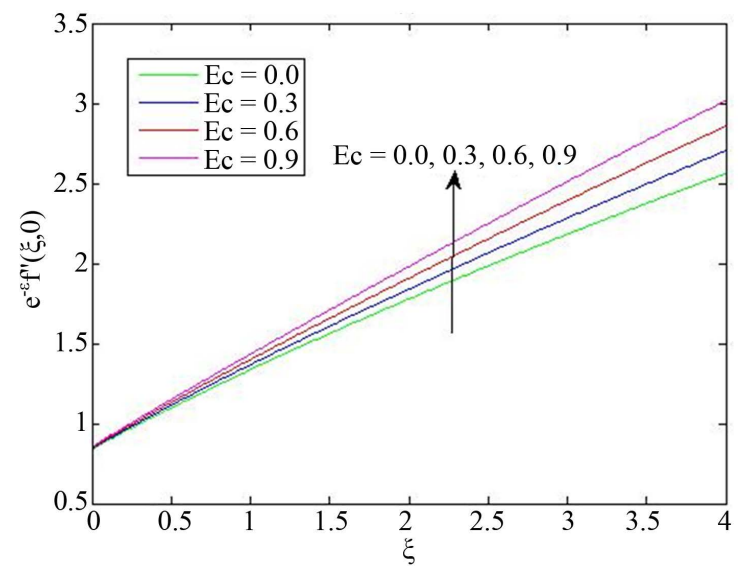

(a)

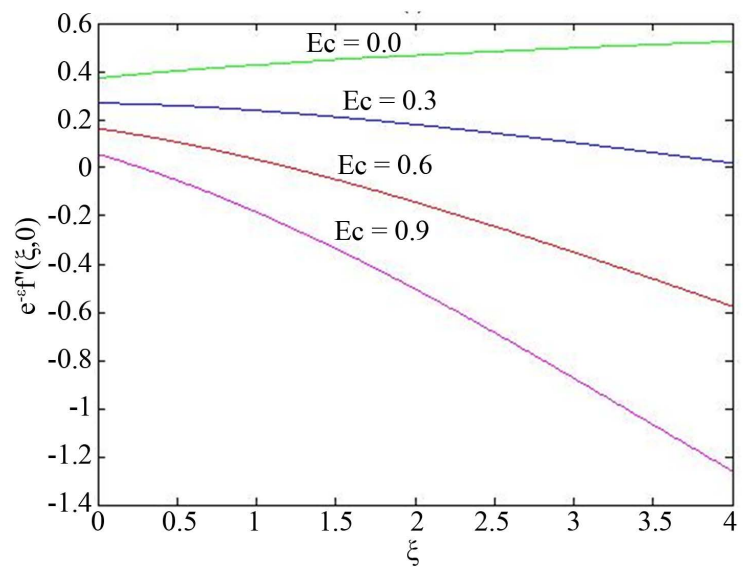

(b)

Figure 5. The variation of (a) skin friction coefficient and (b) Nusselt number with $\xi$ and $E c$ for $S=0.5, n=0.2$, $\varepsilon=-0.3$. 
cient and a decrease in the Nusselt number. The results for the heat transfer coefficient indicate that at a fixed value of $E c>0$ there is a critical value of $\xi$ beyond which the heat transfer coefficient changes sign from positive (heat flows from fluid to the wall) to negative (heat flows from wall to the fluid in the boundary layer).

\section{Conclusions}

A non-Darcy mixed convection boundary layer flow has been investigated. The results have shown that the non-similarity parameter $\xi$, which at particular stream-wise location represents thermal buoyancy, has the effect of accelerating the scaled free-stream velocity, resulting in super-velocities within the boundary layer. This effect is exacerbated by increasing viscous dissipation parameter and the viscosity variation parameter. The temperature dependent viscosity decreases with increase in buoyancy-related parameter and viscosity variation parameter but increases with increase in the viscous dissipation parameter.

The skin friction coefficient increases with increase in the viscosity variation parameter, the viscous dissipation parameter and the buoyancy-related parameter. For flows with viscous dissipation effects, there is a critical value of the buoyancy related parameter, above which the Nusselt number changes sign from positive (wall extracts heat from fluid) to negative (fluid extracts heat from wall).

\section{Acknowledgements}

The author would like to thank the East African Universities Mathematics Programme (EAUMP) for their financial support.

\section{References}

[1] Nield, D.A. and Bejan, A. (2006) Convection in Porous Media. Springer and Business Media, New York.

[2] Pop, I.I. and Ingham, D.B., Eds. (2001) Convective Heat Transfer: Mathematical and Computational Modelling of Viscous Fluids and Porous Media. Elsevier Science \& Technology Books, The Netherlands.

[3] Kaviany, M. (1995) Principles of Heat Transfer in Porous Media. Springer-Verlag, New York. http://dx.doi.org/10.1007/978-1-4612-4254-3

[4] Vafai, K., Ed. (2005) Handbook of Porous Media. Taylor \& Francis, New York.

[5] Karabis, A., Kafoussias, N. and Xenos, M. (1995) Numerical Study of the Combined Forced-Free Convection Boundary Layer Flow through a Highly Permeable Porous Medium. Mechanics Research Communications, 22, 503-510. http://dx.doi.org/10.1016/0093-6413(95)00054-u

[6] Chen, C.H. (1998) Non-Similar Solutions for Non-Darcy Mixed Convection from a Non-Isothermal Horizontal Surface in a Porous Medium. International Journal of Engineering Science, 36, 251-263. http://dx.doi.org/10.1016/S0020-7225(97)00074-8

[7] Vafai, K. and Tien, C.L. (1981) Boundary and Inertia Effect on Flow and Heat Transfer in Porous Media. International Journal of Heat and Mass Transfer, 24, 195-203. http://dx.doi.org/10.1016/0017-9310(81)90027-2

[8] Hong, J.T., Tien, C.L. and Kaviany, M. (1985) Non-Darcy on Vertical Plate Natural Convection in Porous Media with High Porosities. International Journal of Heat and Mass Transfer, 28, 2149-2157. http://dx.doi.org/10.1016/0017-9310(85)90109-7

[9] Hossain, M.A. and Munir, M.S. (2000) Mixed Convection Flow from a Vertical Flat Plate with Temperature Dependent Viscosity. International Journal of Thermal Sciences, 39, 173-183. http://dx.doi.org/10.1016/S1290-0729(00)00237-4

[10] Jayanthi, S. and Kumari, M. (2007) Effect of Variable Viscosity on Non-Darcy Free or Mixed Convection Flow on a Vertical Surface in an Non-Newtonian Fluid Saturated Porous Medium. Applied Mathematics and Computation, 186, 1643-1659. http://dx.doi.org/10.1016/j.amc.2006.08.072

[11] Sparrow, E.M., Quack, H. and Boerner, C.J. (1970) Local Non-Similarity Boundary Layer Solutions. AIAA, 8, 19361942. http://dx.doi.org/10.2514/3.6029

[12] Sparrow, E.M. and Yu, H.S. (1971) Local Non-Similarity Thermal Boundary Layer Solutions. Journal of Heat Transfer, 93, 328-334. http://dx.doi.org/10.1115/1.3449827

[13] Gorla, R.S.R., Bakier, A.Y. and Byrd, L. (1996) Effects of Thermal Dissipation and Stratification on Combined Convection on a Vertical Surface Embedded in a Porous Medium. Transport in Porous Media, 25, 275-282. http://dx.doi.org/10.1007/BF00140984 
[14] Mureithi, E.W. and Mason, D.P. (2010) Local Non-Similarity Solutions for a Forced-Free Boundary Layer Flow with Viscous Dissipation. Mathematical and Computational Applications, 15, 558-573.

[15] Bég, O.A., Bakier, A.Y., Prasad, V.R. and Ghosh, S.K. (2009) Non-Similar Laminar Steady Electrically Conducting Forced Convection Liquid Metal Boundary Layer Flow with Induced Magnetic Field Effects. International Journal of Thermal Sciences, 48, 1596-1606. http://dx.doi.org/10.1016/j.ijthermalsci.2008.12.007 
Scientific Research Publishing (SCIRP) is one of the largest Open Access journal publishers. It is currently publishing more than 200 open access, online, peer-reviewed journals covering a wide range of academic disciplines. SCIRP serves the worldwide academic communities and contributes to the progress and application of science with its publication.

Other selected journals from SCIRP are listed as below. Submit your manuscript to us via either submit@scirp.org or Online Submission Portal.
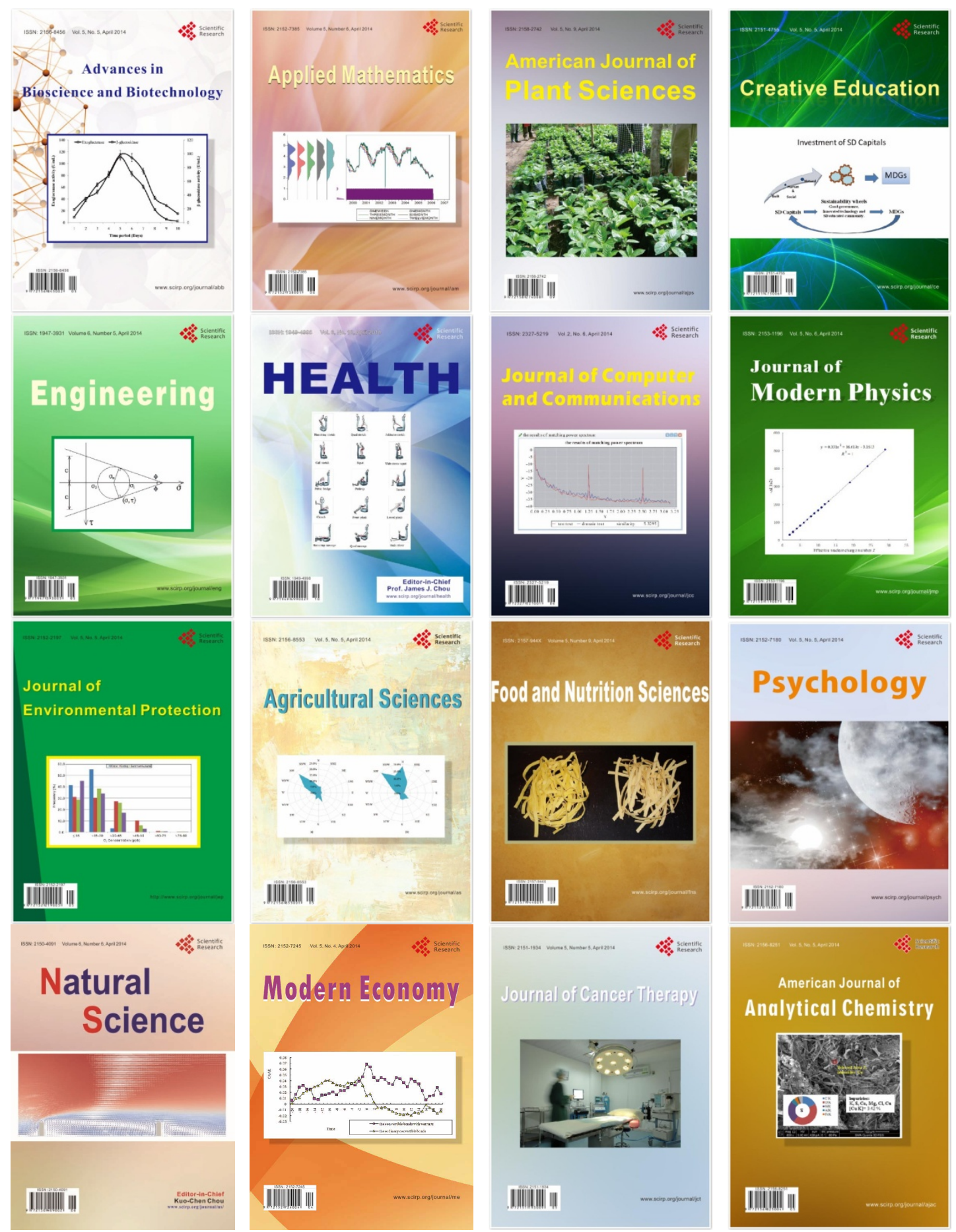